\title{
INDICADOR LÍDER DE LA INVERSIÓN PRIVADA: METODOLOGIAA DE REDES NEURONALES
}

\begin{abstract}
RESUMEN
El presente documento de investigación tiene como objetivo construir un indicador líder de la inversión privada a través del uso de la metodología de redes neuronales artificiales. El indicador líder consiste en el pronóstico de la tasa de crecimiento de la inversión privada para los próximos tres trimestres en base a indicadores adelantados, los cuales se determinan a través de las correlaciones dinámicas de las variables adelantadas con la inversión privada. Es importante mencionar que el aporte de este trabajo de investigación es brindar una metodología de pronóstico, ya que podrían ser relevantes para dar señales sobre el desempeño futuro de la inversión privada y permitirles a los hacedores de política económica tomar decisiones pertinentes.
\end{abstract}

JEL: B22, C13 y E17

PALABRAS CLAVES: Indicador Líder de la Inversión Privada, Redes Neuronales, Pronósticos, Ciclos, Tendencia y Estacionalidad.

\begin{abstract}
This research aims to build a leading indicator of private investment through the use of artificial neural networks methodology. The leading indicator consists of forecaster, the private investment growth rate for the next three quarters based on advanced indicators, which are determined by dynamic correlations between leading indicators and private investment. It is essential to indicate that this research provides a forecast methodology; which would be relevant to provide an indicator of the future performance of private investment and allow policymakers to make relevant decisions.
\end{abstract}

JEL: B22, C13 y E17

KEYWORDS: Leading Indicator to Private Investment, Neural Networks, Forecasting, Cycles, Trend and Seasonality.

\footnotetext{
A Investigador docente de la Escuela Profesional de Economía de la Facultad de Ciencias Contables, Económicas y Financieras de la Universidad de San Martín de Porres y Especialista de Proyecciones Macroeconómicas en la Dirección de Política Macroeconómica y Descentralización Fiscal del Ministerio de Economía y Finanzas de Perú. Se agradece enormemente la asistencia de investigación de Luis Guillermo Del Piélago Alva quien ha realizado un excelente trabajo.
} 


\section{INTRODUCCIÓN}

Los indicadores líderes proporcionan señales de alerta temprana sobre el desempeño de la actividad económica. Dicho de otro modo, pueden anticipar los puntos de inflexión en los ciclos económicos en torno a su crecimiento potencial. En ese sentido, los indicadores líderes son importantes para los hacedores de política económica, ya que les permite formular de forma anticipada instrumentos de política económica para poder contrarrestar la ocurrencia de un evento adverso que podría afectar el normal desempeño de la actividad económica.

En este contexto, dado la importancia de los indicadores líderes en la actividad económica, el presente documento de investigación tiene como objetivo construir un indicador líder de la inversión privada a través de la metodología de redes neuronales. Es importante mencionar que la inversión privada es una variable relevante en la economía peruana, al representar casi el $20 \%$ del PBI; además de que es una variable que genera encadenamientos en otros sectores económicos, es una importante fuente de generación de empleo a través del círculo virtuoso inversiónempleo-consumo.

El resto de la investigación tiene la siguiente estructura. En el apartado 2, se revisa de forma breve el marco teórico vinculado a los indicadores líderes no solo a nivel de la inversión privada sino también a nivel del PBI. El apartado 3 muestra los datos trimestrales de un conjunto de variables que se utilizan para estimar el indicador líder de la inversión, los cuales comprenden desde el primer trimestre de 1994 hasta el primer trimestre de 2019. En el apartado 4 se describe la metodología de redes neuronales $\mathrm{y}$ el filtro de Baxter y King que son relevantes para realizar la estimación del indicador líder de la inversión privada. En el apartado 5, se describen los resultados obtenidos del indicador líder de la inversión. Finalmente, el apartado 5, muestra algunas conclusiones del presente trabajo de investigación.

\section{MARCO TEÓRICO}

El presente trabajo de investigación toma como base de referencia al trabajo realizado por Kapsoli y Bencich (2002), quienes emplean la metodología de redes neuronales para estimar el crecimiento del PBI en función a un conjunto de indicadores adelantados de la actividad económica. En esa misma línea, algunos trabajos de investigación como Loyola
(2009), Mendiburo (2010) y Rodríguez (2010), muestran la relevancia de la inversión privada para determinar la dinámica de la actividad económica y sus componentes no observables. Asimismo, Castellares (2009) hace una revisión de las diferentes metodologías empleadas para el cálculo de los indicadores líderes en Perú y los emplea para detectar el ciclo económico de la economía peruana.

Finalmente, es importante mencionar que la elaboración de indicadores líderes es variada y se estiman a partir de las necesidades particulares que se requieren para la investigación y según lo que se desee pronosticar. En ese sentido, Pérez, Ghurra y Grandez (2016) utilizan el modelo de Aruoba para estimar los quiebres de la tasa de crecimiento del PBI.

\section{DATOS A UTILIZAR}

Para la estimación del modelo se utilizan 12 variables, las cuales deben guardar cierta correlación con la inversión privada. Dichas variables son indicadores adelantados, las cuales se han obtenido de fuentes primarias de información como el Fondo Monetario Internacional - FMI, el Instituto de Estadística e Informática - INEI y el Banco Central de Reserva del Perú - BCRP. Así se pueden mencionar algunas de las variables más relevantes como los términos de intercambio, inversión minera, importaciones de bienes de capital, entre otros. La periodicidad de los datos es trimestral a partir del primer trimestre de 1994 hasta el primer trimestre de 2019. 
Tabla $\mathrm{N}^{\circ} 1$ : Indicadores adelantados de la inversión privada

\begin{tabular}{|l|c|}
\hline \multicolumn{1}{|c|}{ Variables } & Unidad de medida \\
\hline Inflación & Índice \\
\hline Términos de intercambio -TI & Índice \\
\hline Índice de precios de exportación - IPX & Índice \\
\hline Precio del cobre & US\$/lb \\
\hline EMBI & Índice \\
\hline VIX & Índice \\
\hline Inversión pública & Niveles reales a precios de 2007 \\
\hline Inversión minera & Niveles nominales \\
\hline Producción de electricidad & GWh \\
\hline Crédito al sector privado & Niveles nominales (soles) \\
\hline Importaciones de bienes de capital nominales (dólares) \\
\hline Despachos locales de cemento & Toneladas métricas \\
\hline \multicolumn{2}{|c|}{ Inversión privada } \\
\hline \multicolumn{2}{|c|}{ Variable adicional } \\
\hline
\end{tabular}

Fuente: BCRP, INEI, FMI.

Tabla $\mathrm{N}^{\circ}$ 2: Correlaciones dinámicas de los ciclos de la inversión privada y los indicadores adelantados

\begin{tabular}{lcc} 
Variables líderes & $\begin{array}{c}\text { Nivel de adelanto } \\
\text { en el ciclo } \\
\text { (Trimestres) }\end{array}$ & $\begin{array}{c}\text { Correlación } \\
\text { dinámica máxima }\end{array}$ \\
Inversión privada & 0 & 1,000 \\
Inflación & 5 & 0,552 \\
Términos de intercambio & 1 & 0,636 \\
Índice de precios de expotación & 1 & 0,745 \\
Precio del cobre & 6 & 0,634 \\
EMBI & 5 & 0,529 \\
VIX & 3 & 0,376 \\
Inversión pública & 6 & 0,638 \\
Inversión minera & 1 & 0,648 \\
Producción de electricidad & 1 & 0,643 \\
Crédito al sector privado & 3 & 0,763 \\
Importaciones de bienes de capital & 1 & 0,932 \\
Despachos locales de cemento & 1 & 0,924 \\
\hline
\end{tabular}

\section{METODOLOGÍA}

Para la estimación del indicador líder de la inversión privada, es indispensable utilizar el filtro Baxter y King y la metodología de redes neuronales. Particularmente, el filtro de Baxter King (BK) es una aproximación de un filtro de paso de banda lineal, el cual elimina los componentes de tendencia y alta frecuencia (componentes irregulares), teniendo como objetivo mantener los componentes cíclicos (intermedios). El filtro BK descompone una serie de tiempo en tres componentes: una tendencia, un ciclo y un componente irregular.
Donde es la tendencia, es el ciclo y es el componente irregular, Baxter y King (1999).

Una nueva serie de tiempo se genera cuando se aplica una media móvil simétrica finita.

Donde son las constantes o ponderaciones de las observaciones y para $\mathrm{k}=1, \ldots, \mathrm{K}$. Además estas ponderaciones suman cero $=0$

Respecto a la metodología de redes neuronales artificiales, el modelo matemático se construye sobre la base de la neurona funcional como un 
elemento central del sistema nervioso humano cuya tarea es transformar la información de una neurona a la otra. El objetivo del modelo matemático de redes neuronales es el proceso de identificación. En otras palabras, lo que se busca es encontrar una función de entrada y salida; y para que la función de salida pueda encontrar parámetros estimados deseados debe estar sujeta a la minimización de los errores. Los detalles matemáticos se pueden ver en el paper de Kapsoli y Bencich (2002), entre otros.

\section{RESULTADOS}

Los gráficos $\mathrm{N}^{\circ} 1,2$ y 3 , muestran los resultados de la descomposición de la inversión privada en sus componentes estocásticos como el ciclo, tendencia y estacionalidad para el periodo 1T1994 hasta el 1T2019. Particularmente, para obtener el indicador líder de la inversión privada es fundamental hacer este mismo ejercicio para las otras 12 variables y obtener el ciclo. Así, a través de la obtención de los ciclos y haciendo uso de la metodología de redes neuronales, se podrá pronosticar el ciclo de la inversión privada adelantada tres trimestres (ver gráfico $\mathrm{N}^{\circ} 4$ ). Cabe mencionar que la proyección muestra que hay una recuperación del ciclo de la inversión privada entre el 2T2019 y 4T2019.

\section{Gráfico $\mathbf{N}^{\circ} 1$}

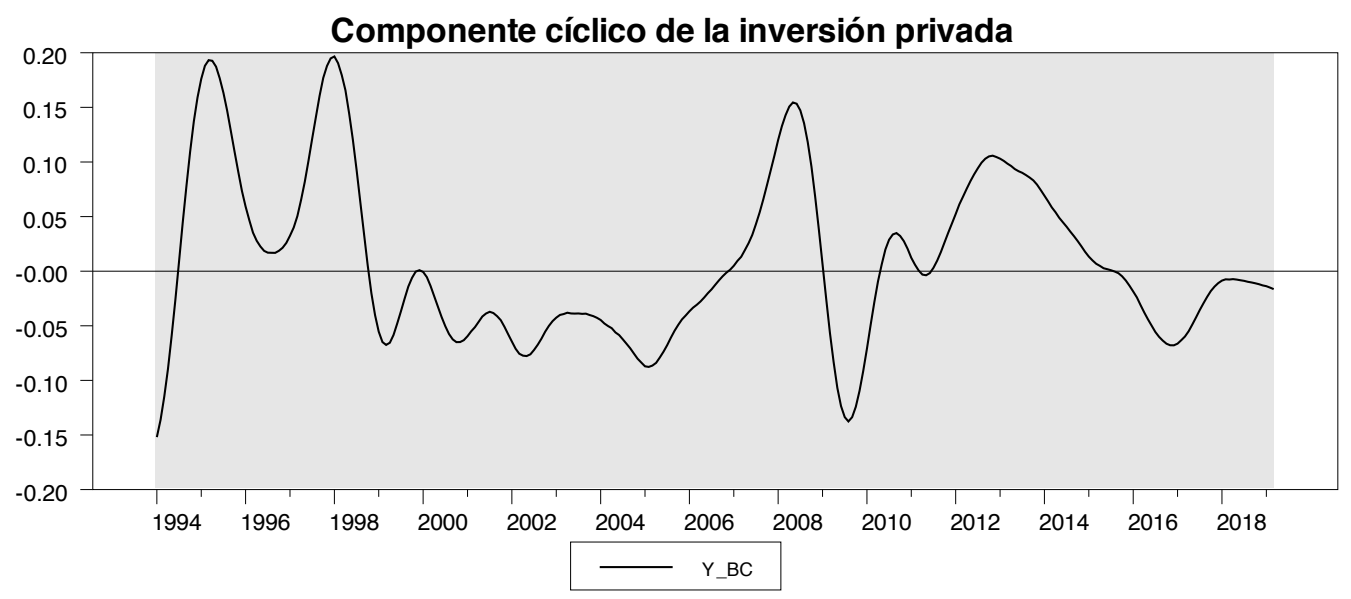

Gráfico $\mathbf{N}^{\circ} 2$

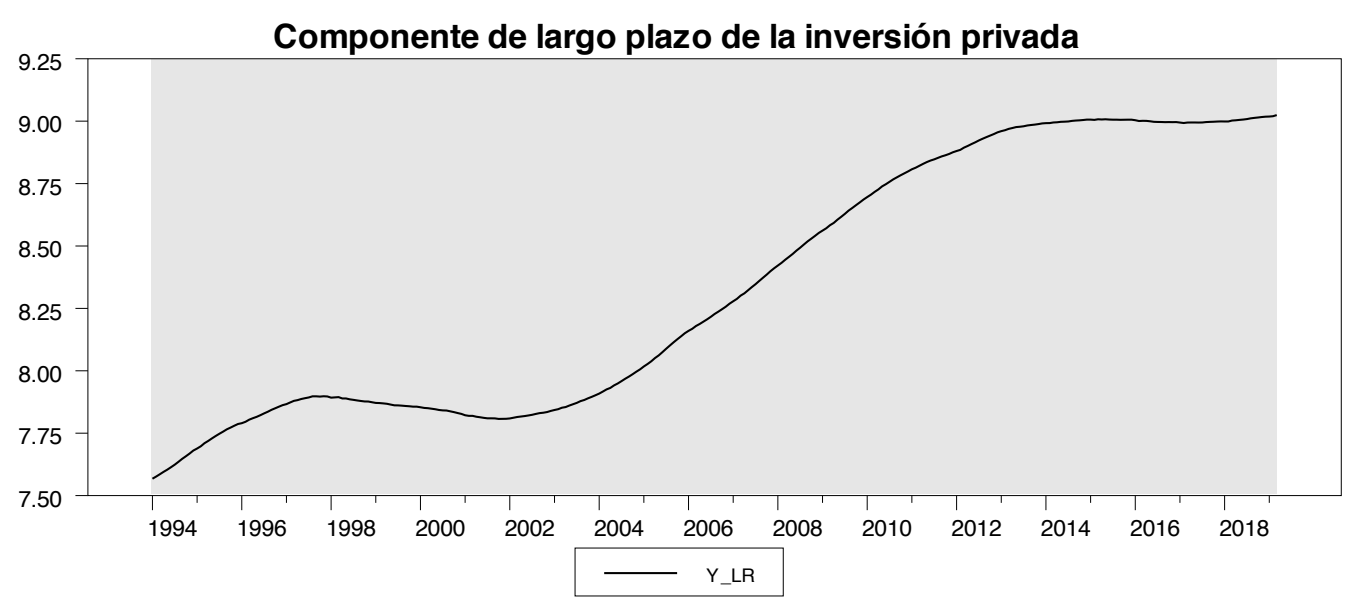




\section{Gráfico $\mathbf{N}^{\circ} 3$}

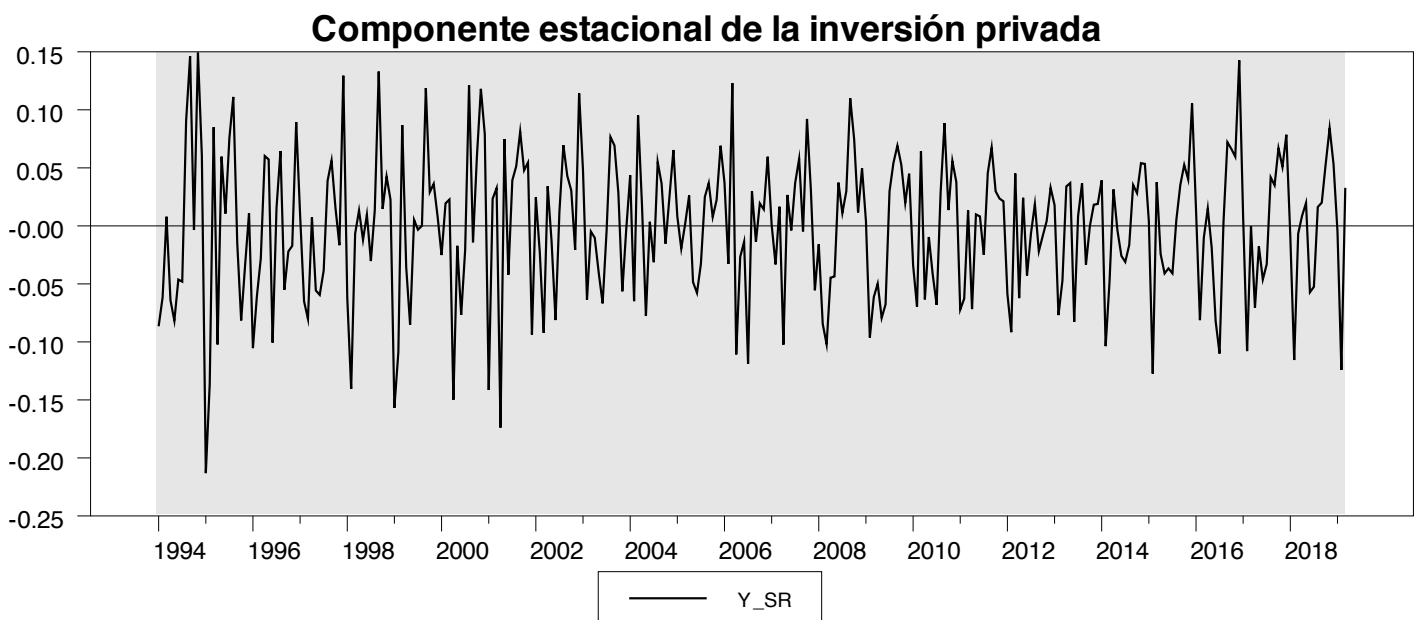

\section{Gráfico $\mathbf{N}^{\circ} 4$}

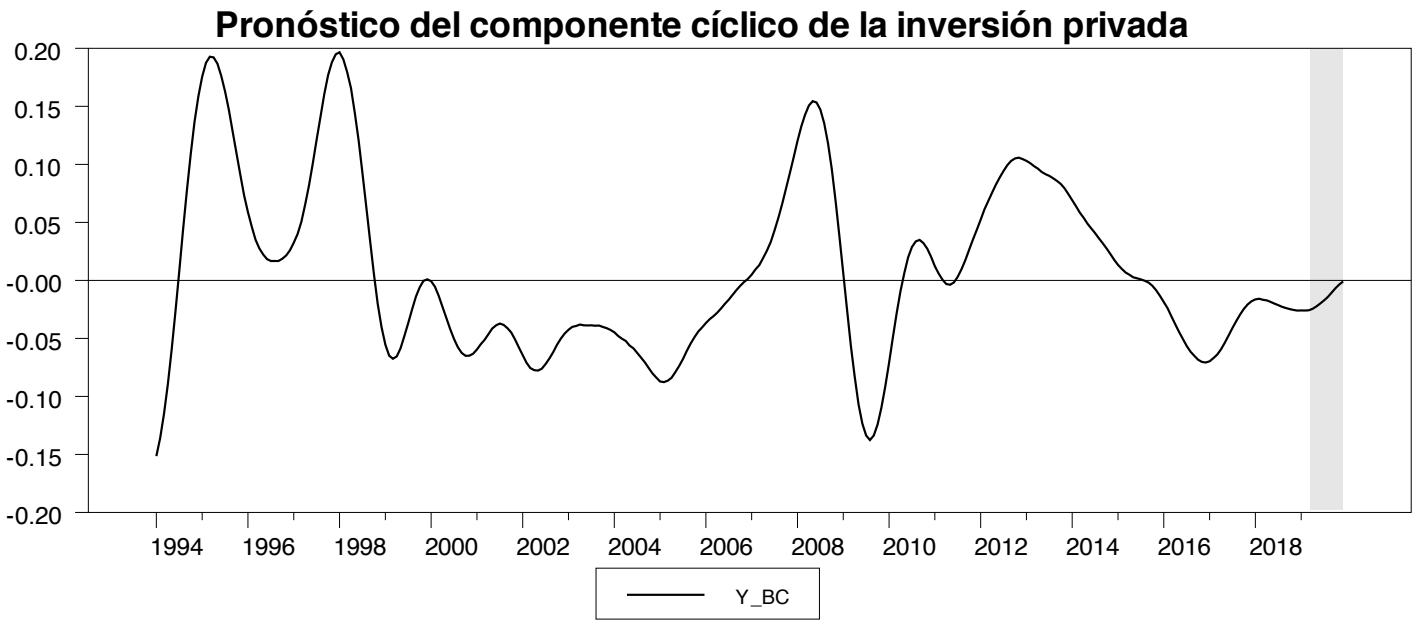

En análisis económico lo que importa es la tasa de crecimiento más que la determinación del ciclo, por lo que se utiliza un modelo ARIMA para proyectar los otros dos componentes (tendencia y estacionalidad). Una vez que se tenga la proyección de los componentes adicionales (gráfico 5 y 6), se realiza el proceso inverso a la descomposición, es decir la agregación de componentes para la determinación de la serie histórica que incluye la proyección de la inversión privada tres trimestres hacia adelante. 


\section{Gráfico $\mathbf{N}^{\circ} 5$}

Pronóstico del componente tendencial de la inversión privada

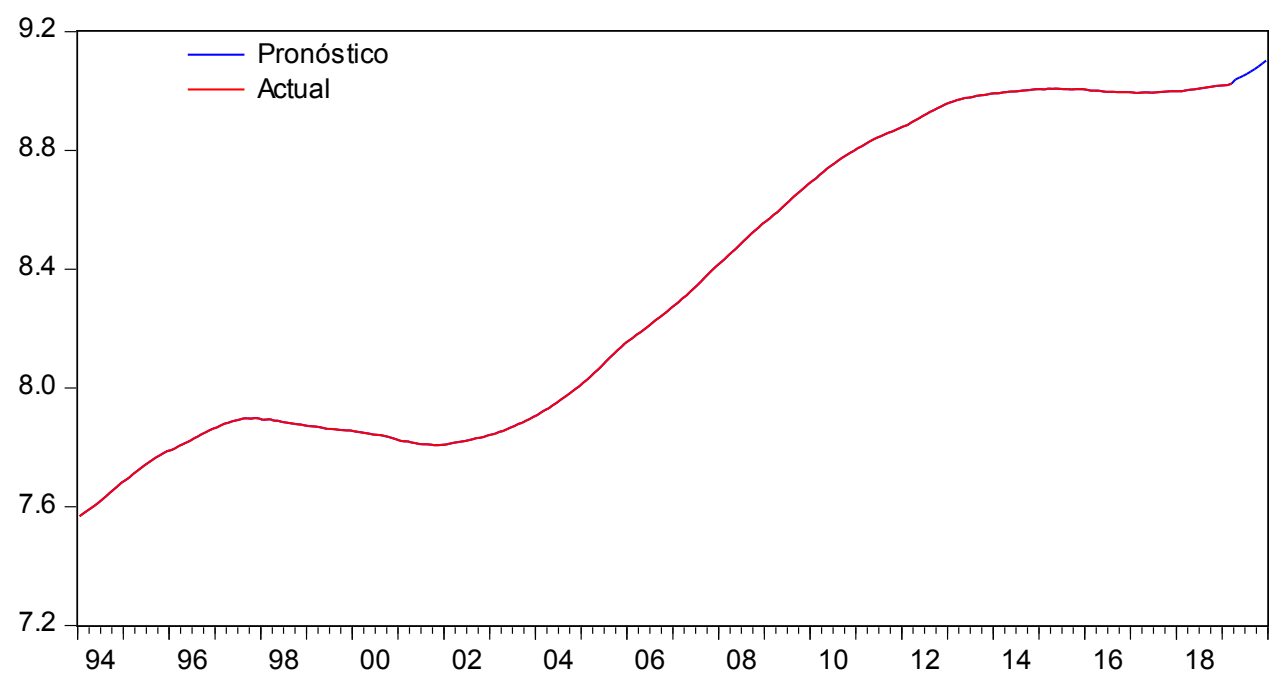

\section{Gráfico $N^{\circ} 6$}

Pronóstico del componente estacional de la inversión privada

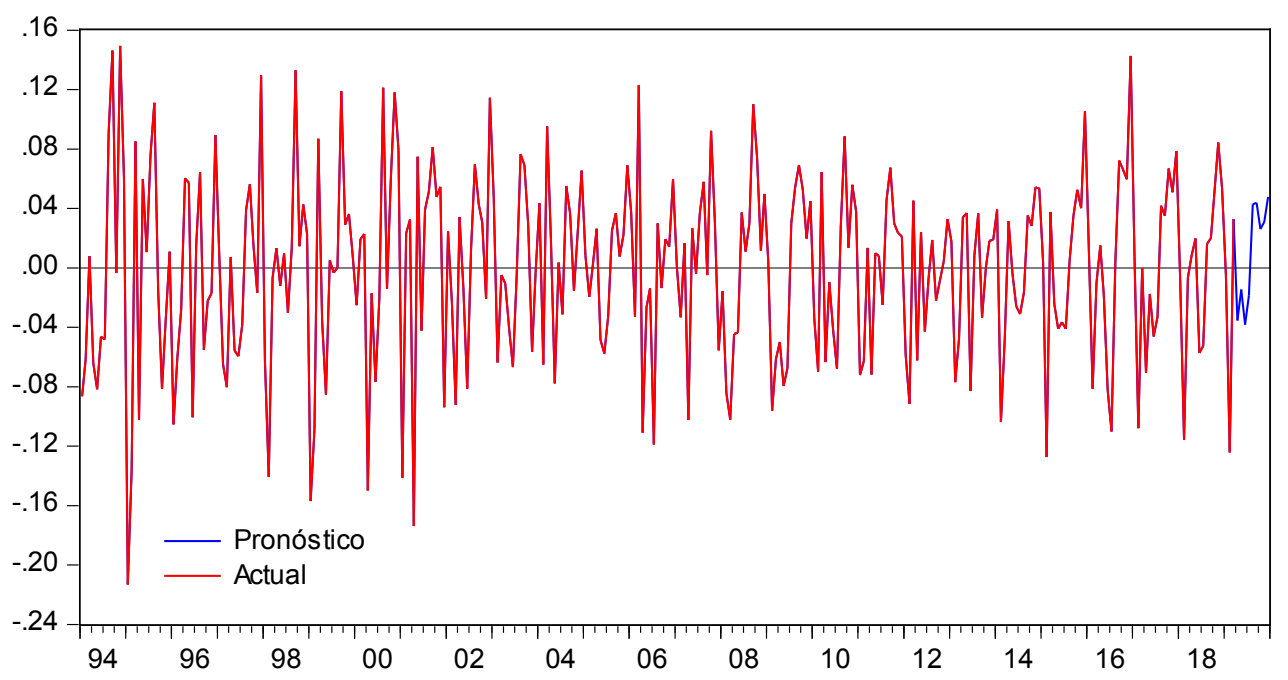

Finalmente, las estimaciones obtenidas a partir de los componentes de la inversión privada no distan de los datos ejecutados. En ese sentido, se han proyectado los datos de la inversión privada para el 2T2019, 3T2019 y 4T2019 (ver gráfico $\mathrm{N}^{\circ} 7$ y tabla $\mathrm{N}^{\circ} 3$ ). Así se puede decir que al cierre de 2019, utilizando la metodología planteada en el presente documento de investigación, la inversión privada crecería $5,2 \%$ con una aceleración importante en la segunda mitad de año (3T2019: 7,8\% y 4T2019:6,2\%). 
Gráfico $\mathbf{N}^{\circ}$ 7: Inversión privada (Var. \% real anual)

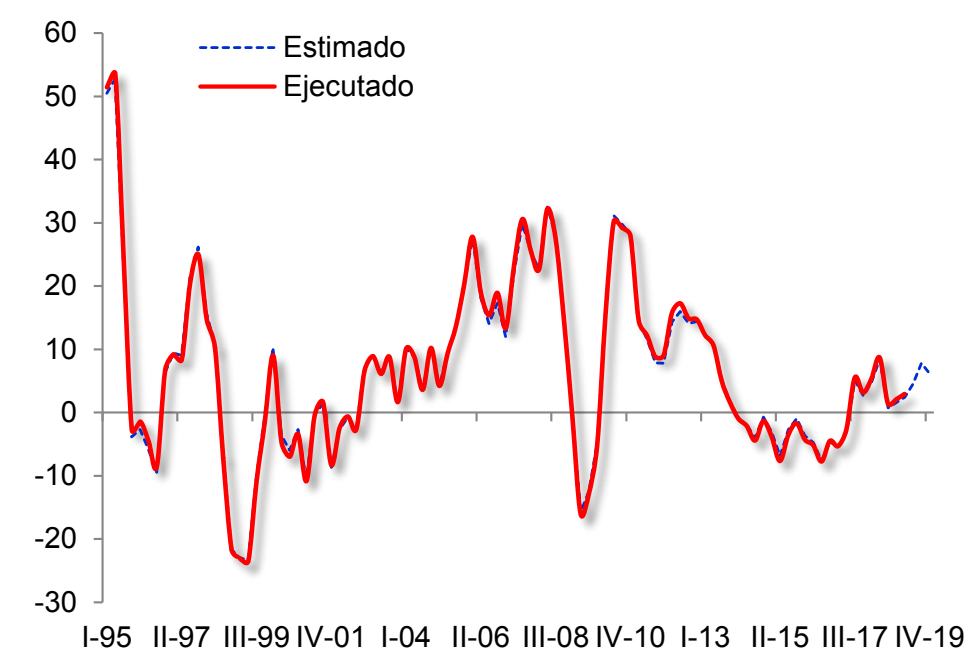

Tabla $\mathrm{N}^{\circ}$ 3: Inversión privada (Var. \% real anual)

\begin{tabular}{lcr}
\hline $\mathbf{2 0 1 7}$ & Ejecutado & Estimado \\
\hline I-17 & $-5,3$ & $-5,6$ \\
II-17 & $-2,6$ & $-3,0$ \\
III-17 & 5,5 & 5,1 \\
IV-17 & 3,1 & 2,7 \\
\hline Año & 0,2 & $-0,2$ \\
\hline \hline $\mathbf{2 0 1 8}$ & Ejecutado & Estimado \\
\hline I-18 & 5,3 & 4,7 \\
II-18 & 8,7 & 8,4 \\
III-18 & 1,4 & 0,7 \\
IV-18 & 2,1 & 1,6 \\
\hline Año & 4,2 & 3,9 \\
\hline \hline $\mathbf{2 0 1 9}$ & Ejecutado & Estimado \\
\hline I-19 & 2,9 & 2,4 \\
II-19 & & 4,5 \\
III-19 & & 7,8 \\
IV-19 & & 6,2 \\
\hline Año & & 5,2 \\
\hline \hline
\end{tabular}

Fuente: BCRP, Estimaciones propias. 


\section{CONCLUSIONES}

El presente trabajo de investigación busca contribuir con una metodología de pronósticos de corto plazo de la inversión privada, al ser esta variable de suma importancia en la actividad económica de Perú.

Asimismo, los pronósticos obtenidos a partir de los indicadores adelantados de la inversión privada, en base a la metodología de redes neuronales, podrían ser relevantes para los hacedores de política económica, ya que les permitiría anticipar el desempeño de esta variable, lo cual podría ser importante para las decisiones de política macroeconómica.

Finalmente, tal como se menciona en la literatura, la metodología planteada se puede mejorar toda vez que los componentes estacionales y tendenciales se estiman a partir de un modelo ARIMA y no por indicadores adelantados.

\section{REFERENCIAS}

[1] Kapsoli, J. y Bencich, B. (2004), Indicadores líderes, redes neuronales y predicción de corto plazo. Revista económica PUCP.

[2] Moron, E., Casas, C., y Carranza, E. (2002), Indicadores líderes para la economía peruana. Universidad del Pacífico.

[3] Pérez, F., Ghurra, O., y Grandez, R. (2016), Un indicador líder de actividad real para Perú. BCRP.

[4] Loyola, J. (2009), Comportamiento de la inversión privada durante los ciclos económicos. BCRP.
[5] Mendiburú, C. (2010), La inversión privada y el ciclo económico en el Perú. BCRP.

[6] Flores, J. (2014), Un modelo de factores dinámicos con expectativas aplicados a un indicador líder para la inversión privada. Tesis PUCP.

[7] Martínez, M., y Quineche, R. (2014), Un indicador lider para el nowcasting de la actividad económica del Perú. BCRP.

[8] Yu, L., Huang, W., Lai, K., y Nakamori, Y. (2007), Neural networks in finance and economics forecasting. International journal of information technology and decision making.

[9] Yalcinoz, T., y Short, M. (1998), Neural networks approach for solving economic dispatch problem with transmission capacity constraints. IEEE, volume 13.

[10] Ntumba, A. (2002), Macroeconomic forecasting: comparison between artificial neuronal networks and econometric model.

[11] Falat, L., y Pancikova, L. (2015), Quantitative Modelling in Economics with Advanced Artificial Neural Networks. Procedia economics and finance. 